\title{
A VECTOR ENERGY FUNCTION APPROACH FOR SECURITY ANALYSIS OF AC/DC SYSTEMS
}

\author{
Christopher L. DeMarco \\ Claudio A. Cañizares \\ Member, IEEE \\ Student Member, IEEE \\ Department of Computer and Electrical Engineering \\ University of Wisconsin-Madison, WI 53706
}

\begin{abstract}
This paper examines dynamic behavior in system models that reflect reasonably detailed (third order) HVDC dynamics along with ac system models that include reactive flows, and frequency and voltage dependent load models. A vector Lyapunov function approach is employed to define a system wide energy function that can be used for general security analysis. The paper describes the derivation of individual component Lyapunov functions for simplified models of HVDC links connected to "infinitely strong" ac systems, along with a standard ac only system Lyapunov function. A novel method of obtaining weighting coefficients to sum these components for the overall system energy function is proposed. Use of the new energy function for transient stability and security analysis is illustrated in a test system. Keywords: HVDC, Lyapunov functions, transient energy functions, transient stability, dynamic security assessment.
\end{abstract}

\section{BACKGROUND AND MOTIVATION}

Use of energy function methods for ac only systems has seen a long history of development in the power systems literature. With several refinements in its application over the last decade, "transient energy function" (TEF) methods are gaining acceptance as a very useful supplement to time domain simulation of individual fault scenarios. To extend the usefulness of such methods further, it is important that the underlying system models be capable of accommodating reasonably detailed representations of important power system components. Clearly, the ability to represent dynamic effects of HVDC links on both transient stability and voltage stability is a useful extension.

Existing literature has provided a foundation for incorporating HVDC models into energy method stability analysis. In [1], the authors describe a scheme for a first order HVDC controller model, representing a single constant current controller. The coupling of these dynamics to a classical model for ac generators is considered. Several system energy functions are examined with the goal of mininizing conservatism in clearing time estimates for fault studies. While the explicit representation of HVDC dynamics is an important step, the model is limited. First, the first order linear model

91 SM 421-8 PWRS A paper recommended and approved by the IEEE Power System Engineering Committee of the IEEE Power Engineering Society for presentation at the IEEE/PES 1991 Summer Meeting, San Diego, California, July 28 - August 1, 1991 . Manuscript submitted January 29, 1991; made available for printing May 17, 1991. cannot represent switching between control modes inherent in ac/ $\mathrm{dc}$ converters. Moreover, the ac system model used assumed constant bus voltage magnitudes throughout, so that reactive power flows and voltage dependent loads could not be represented. This limits the usefulness in examining HVDC ties to weak ac systems, a topic of considerable interest in stability studies. A second approach to considering HVDC links is found in [2]. In this work, the dynamics of the HVDC system are essentially treated as infinitely fast relative to ac system variables, so that the $\mathrm{dc}$ line behavior can be treated by a set of purely algebraic equations. This approach implicitly assumes strong voltage support from the ac system, as a fixed ac converter voltage is used in solving the dc algebraic equations at each time step. While this approach proved successful in examining transient stability problems when the strong voltage support assumption was satisfied, it could prove inaccurate in treating operating conditions where reactive flows and voltage variations are significant $[3,4,5,6]$.

The work presented here builds on the results of [1] and [2] by using a true dynamic model for the HVDC system. Unfortunately, by including such detailed dynamics, standard approaches to constructing a rigorous Lyapunov function ${ }^{1}$ for the full system dynamics becomes analytically intractable. In particular, confirming that a candidate Lyapunov function is non-increasing along any trajectory does not appear feasible for the models examined. Instead, the approach taken here constructs functions which are Lyapunov functions only for de-coupled models of the ac system alone and the dc link alone. One then has an energy function component for the ac system, and one energy function component for each HVDC link. The approach is suited to dealing with systems having multiple dc lines. The overall system wide energy function is constructed as a weighted sum of these components, motivated by vector Lyapunov function concepts as explained in [7]. The weighting coeffcients, denoted by the vector $\xi$, are chosen so that the energy function is locally positive definite at the stable equilibrium point (s.e.p.) and approximately non-increasing along trajectories. We will describe a methodical approach to selecting $\xi$ by enforcing these conditions.

\section{HVDC MODEL}

The HVDC controllers are simulated based on the simple control scheme shown in Fig. 1, and its principal character-

${ }^{1}$ The term Lyapunov function will be used for a scalar function of state that is proven locally positive definite about the stable equilibrium, and non-increasing along any system trajectory. The term "energy function" will be used when the function in question can not be formally proven non-increasing along all possible trajectories 
istics can be reproduced by the control circuit depicted in Fig. 2 [5]. Although these circuits are just a first approximation to the more complicated HVDC control structures, they recreate several of the main properties of actual systems. Voltage-Dependent Current Order Limits (VDCOL) can be readily introduced into this model by representing the controller current order as a nonlinear function of either the dc or ac voltages $[3,4]$.

Equations (1) below are to simulate the HVDC system under these control criteria $[3,4,5,6]$. Here $V_{d_{r}}$ and $V_{d_{i}}$ are the per unit dc terminal voltages at the rectifier and inverter ends, respectively. $I_{n}(\mathrm{kA})$ and $V_{n}(\mathrm{kV})$ are the base quantities for the dc system, and $S_{n}$ is the base power in MVA for the ac side. $X_{c_{r}}$ and $X_{c_{i}}$ are the per unit commutation reactances, and $R_{d}$ and $L_{d}$ are the per unit dc line parameters. The products $a_{r} V_{r}$ and $a_{i} V_{i}$ are the per unit ac bus voltages at the secondary side of the transformers with respect to the dc bus voltage base $V_{n} . S_{r}$ and $S_{i}$ are the per unit magnitudes of the HVDC complex powers at the ac side, and $P_{r}, P_{i}, Q_{r}$ and $Q_{i}$ are the per unit active and reactive powers absorbed by the dc system.

$$
\begin{aligned}
\dot{I}_{d} & =\frac{1}{L_{d}}\left(V_{d_{r}}-V_{d_{i}}\right)-\frac{R_{d}}{L_{d}} I_{d} \\
\dot{x}_{r} & =1_{1}\left(K_{I}\left(I_{o_{r}}-I_{d}\right), x_{r}(t)\right) \\
\dot{x}_{i} & =l_{1}\left(K_{I}\left(I_{d}-I_{o_{i}}\right), x_{i}(t)\right) \\
\cos \alpha_{r} & =I_{2}\left(x_{r}+K_{P}\left(I_{o_{r}}-I_{d}\right)\right) \\
V_{d_{r}} & =\frac{3 \sqrt{2}}{\pi} a_{r} V_{r} \cos \alpha_{r}-\frac{3}{\pi} X_{c_{r}} I_{d} \\
S_{r} & =\frac{V_{n} I_{n}}{S_{n}} \frac{3 \sqrt{2}}{\pi} a_{r} V_{r} I_{d} \\
P_{r} & =\frac{V_{n} I_{n}}{S_{n}} V_{d_{r}} I_{d} \\
Q_{r} & =\sqrt{S_{r}^{2}-P_{r}^{2}} \\
\cos \gamma_{i} & =\mathrm{I}_{2}\left(x_{i}+K_{P}\left(I_{d}-I_{o_{i}}\right)\right) \\
V_{d_{i}} & =\frac{3 \sqrt{2}}{\pi} a_{i} V_{i} \cos \gamma_{i}-\frac{3}{\pi} X_{c_{i}} I_{d} \\
S_{i} & =\frac{V_{n} I_{n}}{S_{n}} \frac{3 \sqrt{2}}{\pi} a_{i} V_{i} I_{d} \\
P_{i} & =-\frac{V_{n} I_{n}}{S_{n}} V_{d_{i}} I_{d} \\
Q_{i} & =\sqrt{S_{i}^{2}-P_{i}^{2}}
\end{aligned}
$$

The controllers are designed to avoid current control overlap. For high rectifier voltages and/or low inverter voltages the rectifier current controller is in operation while the inverter controller is saturated at its minimum value $\gamma_{i_{\text {min }}}$; conversely, for low rectifier voltages and/or high inverter voltages the roles of inverter and rectifier controllers are reversed. During recovery from fault conditions it is typical to have both converters controlling the current for a brief period. This controller "switching" is simulated here by the limit functions $l_{1}(\cdot, \cdot)$ and $l_{2}(\cdot)$, which are defined as follows:

$$
\begin{aligned}
\mathrm{l}_{1}(x, y) & = \begin{cases}x & \text { if } y_{\min }<y<y_{\max } \\
0 & \text { otherwise }\end{cases} \\
\mathrm{l}_{2}(x) & = \begin{cases}x_{\min } & \text { if } x \leq x_{\min } \\
x & \text { if } x_{\min }<x<x_{\max } \\
x_{\max } & \text { if } x \geq x_{\max }\end{cases}
\end{aligned}
$$

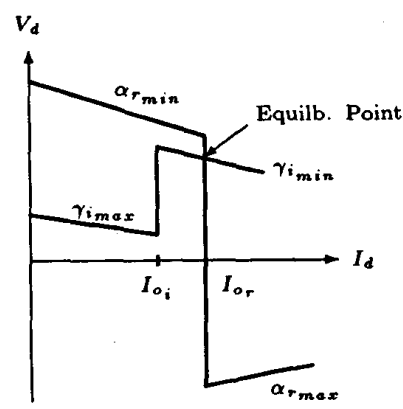

Fig. 1: HVDC control criteria. The rectifier is allowed to go into inverter operation for faster recovery after faults.

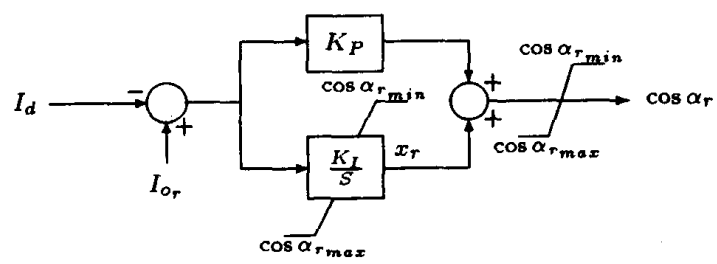

Fig. 2: PI rectifier current controller. The inverter side has a similar control circuit.

Assuming ideal harmonic filtering in the HVDC link, equations (1) are valid to within roughly a $4 \%$ error margin, provided there is a three-valve commutation every $60^{\circ}$ (six-pulse bridge). They are not valid for four-valve commutation, since under these operating conditions the dc link must be represented by a different set of equations [6].

\section{ENERGY FUNCTION FOR DC ONLY}

For fixed ac voltages $V_{r}$ and $V_{i}$ in rectifier or inverter control mode, the dynamics of the current controller are linear. Hence, for each control mode we may construct a state equation and a Lyapunov equation [8] of the form:

$$
\begin{aligned}
\dot{\mathbf{x}}^{c} & =\mathbf{A}^{c} \mathbf{x}^{c}+\mathbf{b}^{c} \\
V_{d c}^{c}\left(\mathbf{x}^{c}\right) & =\frac{1}{2}\left(\mathbf{x}^{c}-\mathbf{x}_{s}^{c}\right)^{T} \mathcal{P}^{c}\left(\mathbf{x}^{c}-\mathbf{x}_{s}^{c}\right) \\
\mathcal{P}^{c} \mathbf{A}^{c}+\mathbf{A}^{c T} \mathcal{P}^{c} & =-I_{2 \times 2} \quad\left(\Rightarrow \dot{V}_{d c}^{c}\left(\mathbf{x}^{c}\right)<0\right)
\end{aligned}
$$

Here $x^{c}=\left[\begin{array}{ll}x_{r} & I_{d}\end{array}\right]^{T}=\mathbf{x}^{r}$ for rectifier current control, or $\mathbf{x}^{c}=\left[\begin{array}{ll}I_{d} & x_{i}\end{array}\right]^{T}=\mathbf{x}^{i}$ for inverter current control, and $\mathbf{x}_{s}^{c}$ is the corresponding stable equilibrium value. $I_{2 \times 2}$ is the $2 \times 2$ identity matrix.

The complete behavior of the two controllers is determined by the limit function which causes a switch between the control modes. The composite state vector in this switching model is $\mathbf{x}=\left[\begin{array}{lll}x_{r} & I_{d} & x_{i}\end{array}\right]^{T}$. To define a candidate Lyapunov function with $\mathcal{P} \in \mathbf{R}^{3 \times 3}$, we combine components of matrices $\mathcal{P}^{r}$ and $\mathcal{P}^{i}$ as shown in (3), where $\beta=\mathcal{P}_{22}^{r} / \mathcal{P}_{13}^{i}$ and $\Delta \mathbf{x}=\mathbf{x}-\mathbf{x} s$.

$$
V_{d c}(\mathbf{x})=\frac{1}{2} \Delta \mathbf{x}^{T} \underbrace{\left[\begin{array}{ccc}
\mathcal{P}_{11}^{r} & \mathcal{P}_{12}^{r} & 0 \\
\mathcal{P}_{12}^{r} & \mathcal{P}_{22}^{r} & \beta \mathcal{P}_{12}^{i} \\
0 & \beta \mathcal{P}_{12}^{i} & \beta \mathcal{P}_{22}^{i}
\end{array}\right]}_{\mathcal{P}} \Delta \mathbf{x}
$$


The time derivative of $V_{d c}(x)$ along trajectories of (1) is examined as two separate cases, i.e.,

- For rectifier control the inverter is saturated at its minimum firing angle $\gamma_{i_{\text {min }}}$, which is its normal operating condition. Here $\Delta x_{i}=0$, and thus

$$
\begin{aligned}
\dot{V}_{d c}(\mathbf{x}) & =\left[\Delta x_{r} \Delta I_{d} 0\right]\left(\mathcal{P} \mathbf{A}+\mathbf{A}^{T} \mathcal{P}\right)\left[\begin{array}{c}
\Delta x_{r} \\
\Delta I_{d} \\
0
\end{array}\right] \\
& =\left[\Delta x_{r} \Delta I_{d}\right]\left(\mathcal{P}^{r} \mathbf{A}^{r}+\mathbf{A}^{r T} \mathcal{P}^{r}\right)\left[\begin{array}{c}
\Delta x_{r} \\
\Delta I_{d}
\end{array}\right] \\
& =-\left[\Delta x_{r} \Delta I_{d}\right]\left[\begin{array}{c}
\Delta x_{r} \\
\Delta I_{d}
\end{array}\right]<0
\end{aligned}
$$

- For inverter control the rectifier is now saturated at its minimum firing angle of $\alpha_{r_{m i n}}$. The function $V_{d c}(\mathbf{x})$ can then be rewritten as

$$
\begin{aligned}
V_{d c}(\mathbf{x})= & \frac{1}{2} \mathcal{P}_{11}^{r} \Delta x_{r}^{2}+\mathcal{P}_{12}^{r} \Delta x_{r} \Delta I_{d} \\
& +\frac{\beta}{2}\left[\Delta I_{d} \Delta x_{i}\right]\left[\begin{array}{c}
\Delta I_{d} \\
\Delta x_{i}
\end{array}\right]
\end{aligned}
$$

where $\Delta x_{r}$ is a positive constant. Then it follows that

$$
\dot{V}_{d c}(\mathbf{x})=\mathcal{P}_{12}^{r} \Delta x_{r} \dot{I}_{d}-\beta\left[\Delta I_{d} \Delta x_{i}\right]\left[\begin{array}{l}
\Delta I_{d} \\
\Delta x_{i}
\end{array}\right]
$$

The structure of the inverter and rectifier equations is such that in general $\beta>0$, and $\mathcal{P}_{12}^{r}<0$. Furthermore, it can be shown from equations (1) that $\dot{I}_{d}>0$, based on the assumption that the ac voltages remain constant. Hence, $\dot{V}_{d c}(\mathbf{x}) \leq 0$ for all system trajectories.

To test how this function behaves when the ac voltages are allowed to change, the ac systems at both rectifier and inverter sides were modelled as fixed voltage sources behind constant reactances. The capacitive voltage support at the ac converter buses was also taken into account. In this case the system equations are nonlinear, and function (3) serves as a candidate local Lyapunov function for the ac/dc system modelled. The positive definiteness of $V_{d c}(\mathbf{x})$ is obvious its behavior along system trajectories must be examined. Several ac balanced faults were simulated on this system by instantaneously changing the Short Circuit Ratio (SCR) at either side of the dc link, and the dc energy function was tracked for different ac system strengths.

Figures 3 and 4 depict the HVDC state variables and the corresponding dc energy function, when an ac fault is simulated by reducing the SCR from 8 to 3 on the rectifier side while the inverter remains at a SCR of 8 . Here one can observe that the "energy", $V_{d c}(\mathbf{x})$, smoothly increases as the system state moves away from the stable equilibrium during the fault conditions, and after clearing the fault, when the system recovers its original state, $V_{d c}(\mathbf{x})$ monotonically decreases towards zero; this is the kind of behavior expected from the system energy. The same energy behavior was observed for several simulations with different rectifier and inverter ac system strengths and faults. In all these cases the overlap angles $\mu_{r}$ and $\mu_{i}$ were traced to detect any changes in converter operation mode, which would indicate the onset of conditions outside the validity of dc model used in this paper.

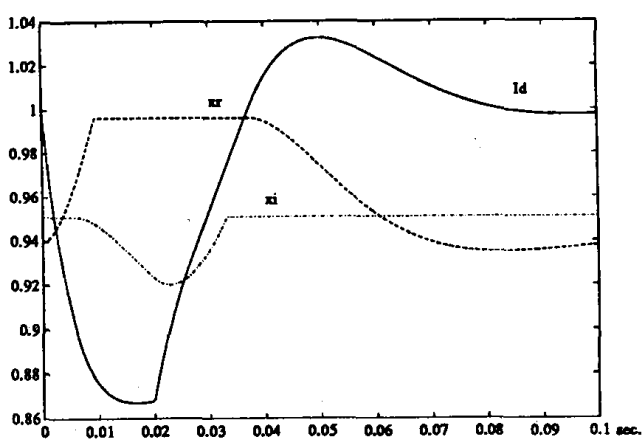

Fig. 3: $H V D C$ state variables for a fault at the ac rectifier side with clearing time of $0.02 \mathrm{sec}$.

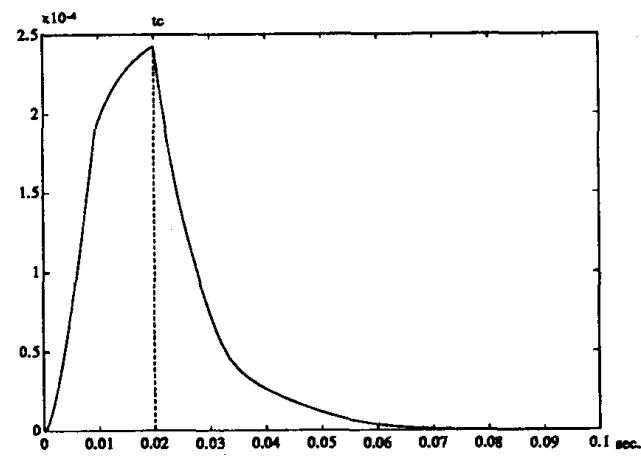

Fig. 4: DC energy function for state variables in Fig. 3.

We also observed that the system equations in this case possess several physically unfeasible equilibria. These were also obtained when solving the power flow equations for the ac/dc system shown in Fig. 6.

\section{ENERGY FUNCTION FOR AC ONLY}

During the last decade, many researches have thoroughly analyzed the use of energy functions for the direct stability assessment of ac networks. Particularly, the TEF for structure preserving ac system models has been widely studied $[9,10,11,13]$, and it has been shown to be defined by

$$
V_{a c}^{a c}=\int_{\left(0, \delta^{0}, V^{0}\right)}^{(\omega, \delta, V)}\left[\begin{array}{lll}
(\mathrm{M} \lambda)^{T} & \mathbf{f}^{T}(\alpha, \mathbf{u}) & \mathbf{g}^{T}(\alpha, \mathbf{u})
\end{array}\right]\left[\begin{array}{l}
\mathrm{d} \lambda \\
\mathrm{d} \alpha \\
\mathrm{d} \mathbf{u}
\end{array}\right]
$$

where $M$ is the diagonal generator inertia matrix, and $f(\delta, V)$ and $g(\delta, V)$ are normalized active and reactive power bus mismatch equations, respectively. For systems with losses, an added constant correction term can account for the transfer conductance losses at the s.e.p., forcing the ac energy function to have a local minimum at this point, as explained in [14]. The ac TEF for an $n$ bus system can be expressed in closed form as:

$$
\begin{aligned}
V_{a c}^{a c}= & \frac{1}{2} \omega^{T} \mathrm{M} \omega+\frac{1}{2} \sum_{k=1}^{n} \sum_{j=1}^{n} B_{k j} V_{k}^{0} V_{j}^{0} \cos \left(\delta_{k}^{0}-\delta_{j}^{0}\right) \\
& -\frac{1}{2} \sum_{k=1}^{n} \sum_{j=1}^{n} B_{k j} V_{k} V_{j} \cos \left(\delta_{k}-\delta_{j}\right)
\end{aligned}
$$




$$
\begin{aligned}
& -\sum_{k=1}^{n} P_{k}\left(\delta_{k}-\delta_{k}^{0}\right)-\sum_{k=1}^{n} \int_{V_{k}^{0}}^{V_{k}} \frac{Q_{k}(\nu)}{\nu} \mathrm{d} \nu \\
& +\sum_{k=1}^{n} \sum_{j=1}^{n} G_{k j} V_{k}^{0} V_{j}^{0} \cos \left(\delta_{k}^{0}-\delta_{j}^{0}\right)\left(\delta_{k}-\delta_{k}^{0}\right) \\
& +\sum_{k=1}^{n} \sum_{j=1}^{n} G_{k j} V_{j}^{0} \sin \left(\delta_{k}^{0}-\delta_{j}^{0}\right)\left(V_{k}-V_{k}^{0}\right)
\end{aligned}
$$

Here $G_{k j}+j B_{k j}$ is the $k j$ term of the bus admittance matrix, $P_{k}$ and $Q_{k}\left(V_{k}\right)$ are the active and reactive powers injected at bus $k$, and $V_{k} \angle \delta_{k}$ is the bus voltage phasor. Here $\omega$ is defined relative to a synchronous reference frame, and the $P_{k}$ 's are corrected to account for the post-fault system equilibrium, as explained in [12].

\section{ENERGY FOR COUPLED AC AND DC}

The next step is to analyze the independent behavior of the $\mathrm{dc}$ and ac energy functions defined above, in an ac/dc system including generator dynamics. Towards that objective, both energy functions were traced for an ac/dc test system during the recovery period from several ac balanced faults and also from sets of arbitrary initial conditions.

The $\mathrm{dc}$ active and reactive powers were treated as special loads of the ac system, similar to the approach used in [2] where the energy function accounts for the active power demands of the dc line. Equation (5) shows the terms that have been added to (4) to account for the active and reactive powers of the HVDC link.

$$
\begin{aligned}
V_{a c}^{d c} & =P_{r}\left(V_{r}, x_{r}, I_{d}\right)\left(\delta_{r}-\delta_{r}^{0}\right)+P_{i}\left(V_{i}, x_{i}, I_{d}\right)\left(\delta_{i}-\delta_{i}^{0}\right) \\
& +\int_{V_{r}^{0}}^{V_{r}} \frac{Q_{r}\left(\nu, x_{r}, I_{d}\right)}{\nu} \mathrm{d} \nu+\int_{V_{i}^{0}}^{V_{i}} \frac{Q_{i}\left(\nu, x_{i}, I_{d}\right)}{\nu} \mathrm{d} \nu
\end{aligned}
$$

The integral expressions in (5) can be found from equations (1), where for the rectifier one has

$$
\begin{aligned}
Q_{r}\left(V_{r}\right) & =\frac{3}{\pi} \frac{V_{n} I_{n}}{S_{n}} \sqrt{\mathrm{a}+\mathrm{b} V_{r}+\mathrm{c} V_{r}^{2}} \\
\mathrm{a} & =-X_{\mathrm{c}_{r}}^{2} I_{d}^{4} \\
\mathrm{~b} & =2 \sqrt{2} a_{r} X_{c_{r}} I_{d}^{3} \cos \alpha_{r} \\
\mathrm{c} & =2 a_{r}^{2} I_{d}^{2}\left(1-\cos ^{2} \alpha_{r}\right)
\end{aligned}
$$

The reactive power at the inverter side can be represented by a similar expression.

The total TEF for the ac/dc system is defined then as

$$
V \triangleq \underbrace{V_{a c}^{a c}+V_{a c}^{d c}}_{V_{a c}}+\xi V_{d c}
$$

where the weighting coefficient $\xi$ is restricted to an interval such that the ac/dc energy function is locally positive definite at the s.e.p. The term $V_{a c}^{a c}$, defined in (4), represents the energy in the ac only system, whereas $V_{a c}^{d c}$, defined in (5), is the coupling energy at the ac/dc converter buses.

Assuming that the ac only system is stable when the HVDC link is replaced by the equivalent active and reactive ac converter power injections at the s.e.p., one can define a "decoupled" ac energy function $\widehat{V}_{a c}$ that is locally positive definite at the corresponding s.e.p. [15], where its Hessian has the following structure:

$$
\left.\nabla^{2} \widehat{V}_{a c}\right|_{0}=\left[\begin{array}{cc}
\mathbf{H}_{1} & \mathbf{H}_{2} \\
\mathbf{H}_{2}^{T} & \widehat{\mathbf{H}}
\end{array}\right]
$$

Hence, since

$$
\left.\nabla^{2} V\right|_{0}=\left[\begin{array}{c|c}
\mathbf{H}_{1} & \mathbf{H}_{2} \quad \mathbf{0} \\
\hline \mathbf{H}_{2}^{T} & \mathbf{H} \\
\mathbf{0} &
\end{array}\right]
$$

a necessary condition for $V$ to be locally positive definite is the positive definiteness of the six by six block shown in (7).

$$
\mathbf{H}=\left[\begin{array}{ccc}
\mathrm{A} & \mathbf{0} & \mathbf{C} \\
\mathbf{0} & \mathbf{B} & \mathbf{D} \\
\mathbf{C}^{T} & \mathbf{D}^{T} & \xi \mathcal{P}^{r}
\end{array}\right]
$$

Assuming that the inverter current controller remains saturated for system trajectories around the s.e.p., matrices $\mathbf{A}$; $B, C$, and $D$ are given by:

$$
\begin{aligned}
& \mathbf{A}=\left[\begin{array}{cc}
Q_{r}^{0}-V_{r}^{02} B_{r r} & -\frac{P_{r}^{0}}{V_{r}^{0}}+\left.\frac{\partial P_{r}}{\partial V_{r}}\right|_{0} \\
-\frac{P_{r}^{0}}{V_{r}^{0}}+\left.\frac{\partial P_{r}}{\partial V_{r}}\right|_{0} & \left.\frac{1}{V_{r}^{0}} \frac{\partial Q_{r}}{\partial V_{r}}\right|_{0}-\frac{Q_{r}^{0}}{V_{r}^{02}}-B_{r r}
\end{array}\right] \\
& \mathbf{B}=\left[\begin{array}{cc}
Q_{i}^{0}-V_{i}^{02} B_{i i} & -\frac{P_{i}^{0}}{V_{i}^{0}}+\left.\frac{\partial P_{i}}{\partial V_{i}}\right|_{0} \\
-\frac{P_{i}^{0}}{V_{i}^{0}}+\left.\frac{\partial P_{i}}{\partial V_{i}}\right|_{0} & \left.\frac{1}{V_{i}^{0}} \frac{\partial Q_{i}}{\partial V_{i}}\right|_{0}-\frac{Q_{i}^{0}}{V_{i}^{0^{2}}-B_{i i}}
\end{array}\right] \\
& \mathbf{C}=\left[\begin{array}{cc}
\left.\frac{\partial P_{r}}{\partial x_{r}}\right|_{0} & \left.\frac{\partial P_{r}}{\partial I_{d}}\right|_{0} \\
\left.\frac{1}{V_{r}^{0}} \frac{\partial Q_{r}}{\partial x_{r}}\right|_{0} & \left.\frac{1}{V_{r}^{0}} \frac{\partial Q_{r}}{\partial I_{d}}\right|_{0}
\end{array}\right] \\
& \mathrm{D}=\left[\begin{array}{cc}
0 & \left.\frac{\partial P_{i}}{\partial I_{d}}\right|_{0} \\
0 & \left.\frac{1}{V_{i}^{0}} \frac{\partial Q_{i}}{\partial I_{d}}\right|_{0}
\end{array}\right]
\end{aligned}
$$

and $\mathcal{P}^{r}$ is the positive definite matrix that comes from the solution of matrix equation (2) for rectifier current control.

Using basic properties of the Euclidean norm, one can readily prove that if $\mathbf{A}$ and $\mathbf{B}$ are symmetric positive definite, then

$$
\begin{aligned}
\mathbf{y}^{T} \mathbf{H y}= & \underbrace{\left[\Delta \delta_{r} \Delta V_{r}\left|\Delta \delta_{i} \Delta V_{i}\right| \Delta x_{r} \Delta I_{d}\right]}_{\mathbf{y}^{T}} \mathbf{H}\left[\begin{array}{l}
\mathbf{y}_{1} \\
\mathbf{y}_{2} \\
\mathbf{y}_{3}
\end{array}\right] \\
= & \mathbf{y}_{1}^{T} \mathbf{A y}_{1}+2 \mathbf{y}_{1}^{T} \mathbf{C y}_{3}+\mathbf{y}_{2}^{T} \mathbf{B} \mathbf{y}_{2}+2 \mathbf{y}_{2}^{T} \mathbf{D y}_{3}+ \\
& \xi \mathbf{y}_{3}^{T} \mathcal{P}^{r} \mathbf{y}_{3} \\
\geq & \sigma_{m}(A)\left\|\mathbf{y}_{1}\right\|^{2}-2 \sigma_{M}(C)\left\|\mathbf{y}_{1}\right\|\left\|\mathbf{y}_{3}\right\|+ \\
& \sigma_{m}(B)\left\|\mathbf{y}_{2}\right\|^{2}-2 \sigma_{M}(D)\left\|\mathbf{y}_{2}\right\|\left\|\mathbf{y}_{3}\right\|+ \\
& \xi \sigma_{m}\left(\mathcal{P}^{r}\right)\left\|\mathbf{y}_{3}\right\|^{2} \\
\geq & \left(\sqrt{\sigma_{m}(A)}\left\|\mathbf{y}_{1}\right\|-\sqrt{\xi \frac{\sigma_{m}\left(\mathcal{P}^{r}\right)}{2}}\left\|\mathbf{y}_{3}\right\|\right)^{2}+ \\
& \left(\sqrt{\sigma_{m}(B)}\left\|\mathbf{y}_{2}\right\|-\sqrt{\xi \frac{\sigma_{m}\left(\mathcal{P}^{r}\right)}{2}}\left\|\mathbf{y}_{3}\right\|\right)^{2}+ \\
& \left(\sqrt{2 \xi \sigma_{m}(A) \sigma_{m}\left(\mathcal{P}^{r}\right)}-2 \sigma_{M}(C)\right)\left\|\mathbf{y}_{1}\right\|\left\|\mathbf{y}_{3}\right\|+ \\
& \left(\sqrt{2 \xi \sigma_{m}(B) \sigma_{m}\left(\mathcal{P}^{r}\right)}-2 \sigma_{M}(D)\right)\left\|\mathbf{y}_{2}\right\|\left\|\mathbf{y}_{3}\right\|
\end{aligned}
$$


The minimum and maximum singular values $\left(\sigma_{m}\right.$ and $\sigma_{M}$ respectively) of matrices $A, B, C$, and $D$, considering that $I_{d}^{0}=1 \mathrm{pu}$ and $V_{\mathrm{r}}^{0} \approx 1 \mathrm{pu}$, can be shown to satisfy

$$
\begin{aligned}
& \sigma_{m}(A) \approx-B_{r r}-R_{c_{r}} \frac{P_{r}^{0}}{Q_{r}^{0}}>0 \\
& \sigma_{m}(B) \approx-B_{i i}+R_{c_{i}} \frac{P_{i}^{0}}{Q_{i}^{0}}>0 \\
& \sigma_{M}(C)<\sqrt{\left(1+\frac{P_{r}^{02}}{Q_{r}^{02}}\right)\left[n_{r}^{2}+Q_{r}^{02}+\left(R_{c_{r}}+K_{P} n_{r}\right)^{2}\right]} \\
& \sigma_{M}(D) \approx \sqrt{\left(1+\frac{P_{i}^{02}}{Q_{i}^{02}}\right)\left(Q_{r}^{02}+R_{c_{r}}^{2}\right)}
\end{aligned}
$$

where

$$
\begin{aligned}
R_{c_{r}} & =\frac{V_{n} I_{n}}{S_{n}} \frac{3}{\pi} X_{c_{r}} \\
R_{c_{i}} & =\frac{V_{n} I_{n}}{S_{n}} \frac{3}{\pi} X_{c_{i}} \\
n_{r} & =\frac{V_{n} I_{n}}{S_{n}} \frac{3 \sqrt{2}}{\pi} a_{r}
\end{aligned}
$$

Therefore, the ac/dc TEF, $V$, can be locally positive definite only if the following inequalities hold:

$$
\begin{aligned}
\xi>\frac{2\left[n_{r}^{2}+Q_{r}^{02}+\left(R_{c_{r}}+K_{P} n_{r}\right)^{2}\right]\left(1+\frac{P_{r}^{02}}{Q_{r}^{02}}\right)}{\sigma_{m}\left(P^{r}\right)\left(-B_{r_{r}}-R_{c_{r}} \frac{P_{r}^{0}}{Q_{r}^{0}}\right)} \\
\xi>\frac{2\left(Q_{i}^{02}+R_{c_{r}}^{2}\right)\left(1+\frac{P_{i}^{0^{2}}}{Q_{i}^{0^{2}}}\right)}{\sigma_{m}\left(P^{r}\right)\left(-B_{i i}+R_{c_{i}} \frac{P_{i}^{0}}{Q_{i}^{0}}\right)}
\end{aligned}
$$

These inequalities represent a necessary but not sufficient condition for local positive definiteness of $V$; however, due to the highly diagonal dominant structure of matrix $\mathbf{H}_{1}$ in (6), one can reasonably expect the minimum eigenvalues of $H_{1}$ and $H$ to dominate the maximum singular value of matrix $\mathbf{H}_{2}$. For the sake of completeness, an additional numerical test of positive definiteness of $\left.\nabla^{2} V\right|_{0}$ is performed after the candidate value of $\boldsymbol{\xi}$ has been chosen using the technique described below. Notice also that (8) and (9) primarily depend on the dc power levels at the s.e.p. and the design parameters of the $\mathrm{dc}$ line. This will prove helpful in determining energy profiles for voltage instability studies for the sample system, as shown in the next. section.

The inequalities above yield a minimum value for the coefficient $\boldsymbol{\xi}$ so that one requirement for the ac/dc TEF can be fulfilled. However, a second property desired of the system energy function is that it be decreasing along trajectories. In standard vector Lyapunov function analysis this problem is resolved by using comparison functions [7]. Unfortunately, the analytic complexity of the time derivative for the proposed energy function makes the comparison function approach intractable. Instead a new computational approach is developed. Figure 5 clearly shows that the ac and dc TEF's present opposite behavior, suggesting as an interchange of "energy" between the ac and the dc systems. The same behavior was observed during the post-fault period for

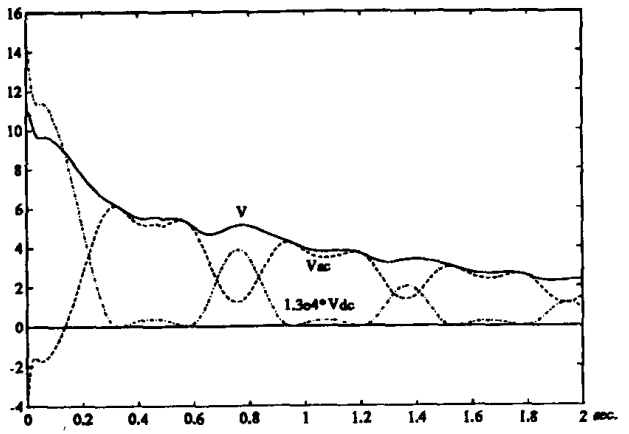

Fig. 5: Total TEF and $a c$ and dc energy functions along the trajectory between the closest, and unique, unstable equilibrium point (u.e.p.) and the s.e.p. for the sample system.

a wide range of ac faults and various system strengths. The choice of $\xi$ can be further constrained by imposing the restriction of nonincreasing behavior along trajectories at a sequence of sampling times, i.e.,

$$
\begin{aligned}
V\left(t_{j}\right) & \geq V\left(t_{j+1}\right) \\
\Rightarrow \xi_{j} & \geq \frac{V_{a c}\left(t_{j+1}\right)-V_{a c}\left(t_{j}\right)}{V_{d c}\left(t_{j}\right)-V_{d c}\left(t_{j+1}\right)}
\end{aligned}
$$

The average value produced by (10), subject to (8) and (9), is selected and tested to see whether the Hessian of the ac/dc TEF at the s.e.p. is positive definite. Initial experience indicates the constraints on $\xi$ will be more easily satisfied in larger systems, where a smaller percentage of ac buses are tightly coupled to the dc system. In constructing the set of $\xi$ values to be averaged, a maximum threshold is set and all values greater than this limit are discarded. This is necessary because the dc energy function applied to the total system can pass near zero at some sample points, causing roundoff errors in evaluating the denominator of (10). The resulting ac/dc energy function $V$ is shown in Fig. 5 for the u.e.p.-s.e.p. trajectory in the test system. Notice that it decreases towards zero as the system trajectory approaches the s.e.p.; however, there are brief time intervals where the energy function actually experiences a relatively small increase. Similar behavior was observed for several ac balanced faults and rectifier system strengths, especially when the system trajectory originates far from the stable equilibrium. The next section shows possible applications of this energy function as a means of assessing the stability of the ac/dc network.

\section{APPLICATION TO SAMPLE SYSTEM}

Figure 6 shows the sample ac/dc system used to simulate several ac balanced faults and voltage collapse scenarios throughout this paper. This system is intended to roughly approximate some of the characteristics of power systems in the western part of the United States. Generator $G_{2}$ supports the voltage at the intermediate load bus, and bus 1 has relatively strong voltage support from generator $G_{1}$. Two different effective short circuit ratios (ESCR) at bus 1, 21.4 (practically an infinite bus) and 6.2, are used to simulate different system strengths, whereas the ESCR at the inverter 


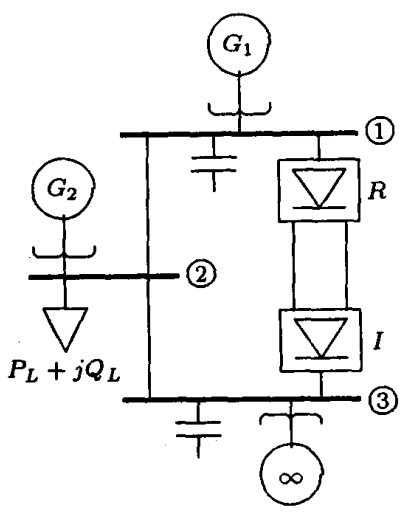

Fig. 6: Sample ac/dc system

side is kept constant at about 4. The dc line is designed to supply approximately two thirds of the power needed at bus 3 , and its rectifier and inverter controllers limits are: $\alpha_{r_{\min }}=5^{\circ}, \alpha_{r_{\max }}=120^{\circ}, \gamma_{i_{\min }}=18^{\circ}$, and $\gamma_{i_{\max }}=40^{\circ}$ $\left(\alpha_{i_{\max }} \approx 120^{\circ}\right)$. The active power for the load at bus 2 is assumed frequency dependent, whereas the reactive power is modelled as a constant reactance (voltage dependent). The generators are simulated as voltage sources behind transient reactance, while the mechanical system is represented using the classical second order swing equations.

Two types of studies were done on the sample network to assess the usefulness of the proposed ac/dc TEF. First, three different faults were applied to this system to compare the critical clearing times obtained by time simulation to those obtained using the ac/dc energy function. AC faults at the rectifier and inverter were represented by reducing the ESCR of the ac converter side, whereas at bus 2 a balanced three-phase fault was directly applied. Second, the energy function was used to determine system proximity to voltage collapse, as suggested in $[14,16]$, when either active power or reactive power is increased at the load bus.

For a rectifier ESCR of 21.4, inequalities (8) and (9), and condition (10) applied to the closest u.e.p.-s.e.p. system trajectory, yielded a weighting coefficient of $\xi=1.3 \times 10^{4}$, for both power load levels shown in table 1. For the ESCR of 6.2 , the coefficient value produced by the proposed method was $\xi=3.15 \times 10^{4}$. Notice that the different load levels did not alter the value of $\xi$, since the HVDC variables are not significantly affected by changes at the load bus.

Table 1 depicts the clearing times for different balanced ac faults simulated in the test system. Two distinct techniques were used to determine the critical clearing times by means of the ac/dc TEF, namely, the controlling u.e.p. and the Potential Energy Boundary Surfaces (PEBS). (Refer to [17] for a succinct explanation of these methods.) Since the modelling of the system yields algebraic constraints that change during the simulation due to system modifications, i.e., applying and clearing faults, the fault trajectories have to be "projected" back to the post-fault system structure. This process simply takes the system state at each point along the fault-on trajectory and computes the corresponding values for implicit variables that would result if the fault were cleared at that instant. The energy value is calculated

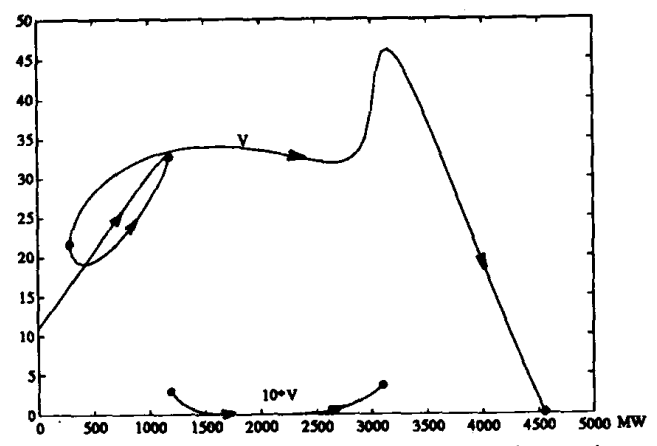

Fig. 7: TEF at various unstable equilibria for active power changes at the load bus. The rectifier ESCR is 21.4 .

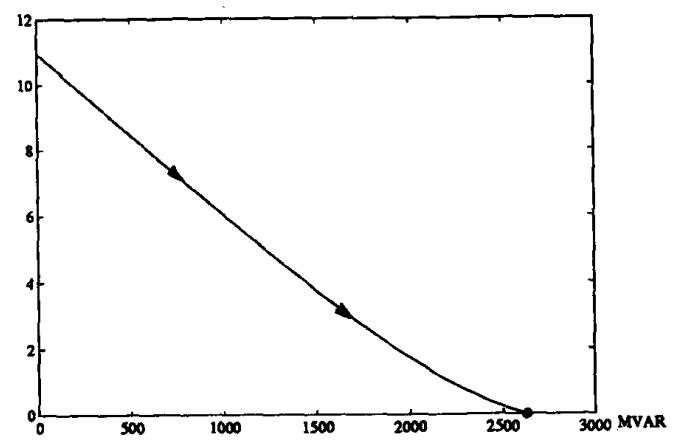

Fig. 8: $T E F$ at various unstable equilibria for reactive power changes at the load bus. The rectifier ESCR is 21.4.

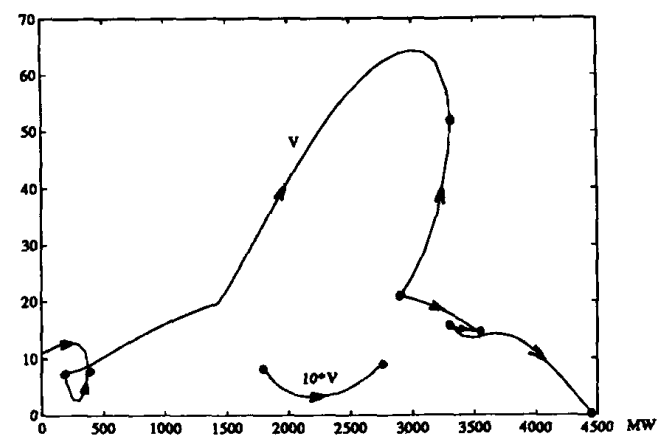

Fig. 9: TEF at various unstable equilibria for active power changes at the load bus. The rectifier ESCR is 6.2.

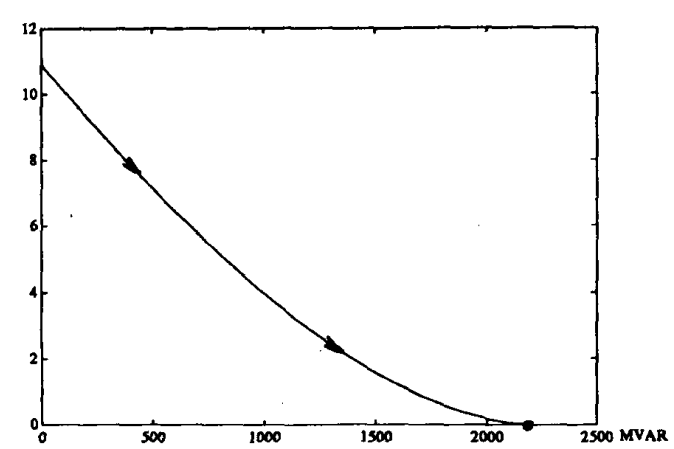

Fig. 10: TEF at various unstable equilibria for reactive power changes at the load bus. The rectifier ESCR is 6.2. 


\begin{tabular}{|c|c|c|c|c|c|}
\hline $\begin{array}{l}\text { ESCR } \\
\text { rectif. }\end{array}$ & $\begin{array}{c}P_{L} \\
\mathrm{MW}\end{array}$ & $\begin{array}{l}\text { Fault } \\
\text { BuS }\end{array}$ & $\begin{array}{l}\text { Time } \\
\text { Simul. }\end{array}$ & $\begin{array}{c}\text { Controll. } \\
\text { u.e.p. }\end{array}$ & $\overline{\text { PEBS }}$ \\
\hline \multirow{6}{*}{21.4} & \multirow{3}{*}{475} & 1 & $\begin{array}{l}0.028^{f} \\
0.030^{\ddagger}\end{array}$ & 0.0092 & - \\
\hline & & 2 & $\begin{array}{l}0.028 \\
0.030\end{array}$ & 0.0067 & 0.029 \\
\hline & & 3 & $\begin{array}{c}0.022 \\
0.023^{5}\end{array}$ & 0.0181 & - \\
\hline & \multirow{3}{*}{2975} & 1 & $\begin{array}{l}0.035 \\
0.038\end{array}$ & 0.0262 & 0.05 \\
\hline & & 2 & $\begin{array}{l}0.0040 \\
0.0045\end{array}$ & 0.0024 & 0.0043 \\
\hline & & 3 & $>0.1^{\top}$ & $>0.1$ & 0.03 \\
\hline \multirow{3}{*}{6.2} & \multirow{3}{*}{475} & 1 & $\begin{array}{l}0.024 \\
0.026\end{array}$ & 0.0072 & - \\
\hline & & 2 & $\begin{array}{l}0.028 \\
0.030\end{array}$ & 0.0072 & 0.029 \\
\hline & & 3 & $\begin{array}{c}0.0158 \\
0.0159^{\S}\end{array}$ & 0.0127 & - \\
\hline
\end{tabular}

Table 1: Critical clearing times (in seconds) from TEF and time simulations for the test system.

based on this choice of implicit variable values. The controlling u.e.p. method underestimates the values of the clearing times, sometimes by an order of magnitude. This is also a problem in tightly interconnected ac only systems with just one u.e.p. neighboring the s.e.p., where differences of a factor of 10 between the simulated clearing times and those predicted by the controlling u.e.p. method are also possible (e.g., for a 5 bus ac system from [18] with 2 generators and 2 loads, the simulated critical clearing time for a solid bus fault is $t_{c c}^{s i m}=0.0495 \mathrm{sec}$, whereas the predicted value using the controlling u.e.p. method yields $t_{c c}^{u . e . p .}=0.002 \mathrm{sec}$.). The PEBS technique sometimes yields better estimates for ac faults away from the dc converters; however, it fails in some cases for the ac/dc system. Note that in two cases the energy methods predict a fault at bus 2 to be more severe than a corresponding fault at bus 3 , which seems inconsistant with the clearing times obtained by simulation. However, the "clearing time" for bus 3 in table 1 does not correspond to a true unstable mode, but represents the instant when the dc inverter enters into four-valve commutation rendering the dc dynamic equations presented in this paper inaccurate [6]. More simulations in larger systems are necessary before drawing definite conclusions, since the authors believe that the PEBS method applied to the proposed ac/dc TEF will produce good clearing time estimates for faults at least one or two buses away from the HVDC link (results in [2] support this idea).

In general, the results above suggest that the value of the proposed TEF at the closest u.e.p. gives a relatively good notion of the dynamic stability of the ac/dc system. This idea can be used to evaluate the vulnerability of the system to voltage collapse, as proposed in [14] and [16] for ac only systems. Figures 7 through 10 show the value of the ac/dc energy function $V$ for all possible system u.e.p.'s; the arrows in the graphs indicate how the equilibria bifurcate as the load power increases. Figures 8 and 10 present a linear behavior similar to that presented in $[16,14]$, which apparently is related to the "quadratic" shape of the "nose" curves (voltage profiles or bifurcation diagrams) that are shown in
[19]. However, Figs, 7 and 9 exhibit a much more complicated structure. Here the energy function for a specific unstable equilibrium has been scaled; this u.e.p. is associated to a second stable equilibrium corresponding to inverter current control that bifurcates twice with the u.e.p. Eventually the system model loses all power flow solutions when the TEF becomes zero (in the literature this is known as the point of voltage collapse). The reader is referred to [19] for an examination of saddle-node bifurcation phenomena in dynamic models of ac/dc systems.

\section{CONCLUSIONS}

This paper has introduced a new method of determining vector energy functions for power system models with HVDC links. The proposed technique for choosing the weighting factor that couples the ac and dc energy functions is thoroughly studied, and the analytic limitations imposed by the complexity of the mathematical models used to represent the HVDC links are explored. The resulting ac/dc TEF, together with the projection of the fault trajectories into the manifold formed by the post-fault algebraic constraints, is utilized in a sample system for transient stability assessments. Initial results appear promising, although additional testing in larger systems is needed.

A possible computational shortcoming is the need for running a complete time simulation of the ac/dc system in order to determine the weighting coefficient. However, the results presented in this paper suggest that unless the stable operating conditions of the HVDC system are significantly changed, the factor $\xi$ will not change for different system structures of the ac network, reducing the need for repeated simulations to determine the closest u.e.p.-s.e.p. system trajectory.

\section{ACKNOWLEDGEMENTS}

The support of this work by EPRI (grant RP-2674-4) and NSF (grant ECS-8857019) is gratefully acknowledged.

\section{REFERENCES}

[1] M. A. Pai, K. R. Padiyar, C. Radhakrishna, "Transient Stability of Multi-Machine AC/DC Power Systems Via EnergyFunction Method," IEEE Trans. Power Apparatus and Systems, Vol. 100, No. 12, Dec. 1981, pp. 5027-5035.

[2] Yi-Xin Ni, A. A. Fouad, "A Simplified Two-Terminal HVDC Model and its use in Direct Transient Stability Assessment," IEEE Trans. Power Systems, Vol. 2, No.4, Nov. 1987, pp. 1006-1012.

[3] Methodology of Integration of HVDC Links in Large AC Systems-Phase 1: Reference Manual, EPRI EL-3004, March 1983.

[4] Methodology of Integration of HVDC Links in Large AC Systems-Phase 2: Advanced Concepts, EPRI EL-4365, Vol. 1, April 1987.

[5] J. Arrillaga, High Voltage Direct Current Transmission, Peter Peregrinus Ltd., London, UK, 1983.

[6] J. Arrillaga, C. P. Arnold, B. J. Harker, Computer Modelling of Electrical Power Systems, John Wiley \& Sons, UK, 1983.

[7] M. A. Pai, Power System Stability Analysis by the Direct Method of Lyapunov, North-Holland, 1981. 
[8] M. Vidyasagar, Nonlinear Systems Analysis, Prentice-Hall, 1978.

[9] A. R. Bergen, D. J. Hill, "A Structure Preserving Model for Power System Stability Analysis," IEEE Trans. Power Apparatus and Systems, Vol. 100, No. 1, January 1981, pp. 25-35.

[10] N. Narasimhamurthi, M. R. Musavi, "A Generalized Energy Function for Transient Stability Analysis of Power Systems," IEEE Trans. Circuits and Systems, Vol. 31, No. 7, July 1984, pp. 637-645.

[11] A. Toslas, A. Aropastathis, P. Varaiya, "A Structure Preserving Energy Function for Power System Transient Stability Analysis," IEEE Trans. Circuits and Systems, Vol. 32, No. 10, October 1985, pp. 1041-1049.

[12] D. J. Hill, "On the Equilibria of Power Systems with Nonlinear Loads," IEEE Trans. Circuits and Systems, Vol. 36 , No. 11, November 1989, pp. 1458-1463.

[13] C. L. DeMarco, A. R. Bergen, "A Security Measure for Random Load Disturbances in Nonlinear Power System Models," IEEE Trans. Circuits and Systems, Vol. 34, No. 12, December 1987, pp 1546-1557.

[14] T. J. Overbye, C. L. DeMarco, "Voltage Security Enhancement Using Energy Based Sensitivities," IEEE/PES Summer Meeting paper 90 SM $478-8$ PWRS, July 1990.

[15] C. L. DeMarco, "A New Method of Constructing Lyapunov Functions for Power Systems," Proc. ISCAS'88, 1988, pp. 905-908.

[16] C. L. DeMarco, T. J. Overbye, "An Energy Based Security Measure for Assessing Vulnerability to Voltage Collapse," IEEE Trans. Power Systems, Vol. 5, No. 2, May 1990, pp. 419-427.

[17] P. Varaiya, F. F. Wu, R. L. Chen, "Direct Methods for Transient Stability Analysis of Power Systems: Recent Results," Proc. of the IEEE, Vol. 73, No. 12, December 1985, pp. 1703-1715.

[18] M. A. Pai, Computer Techniques in Power System Analysis, Tata McGraw-Hill, New Delhi, 1979.

[19] C. A. Cañizares, F. L. Alvarado, C. L. DeMarco, I. Dobson, W. F. Long, "Voltage Collapse and Transient Energy Function Analyses of AC/DC Systems," Report ECE-90-7, Department of Electrical and Computer Engineering, University of Wisconsin-Madison, Nov, 1990.

Christopher L. DeMarco (S'80, M'85) was born in Derby, Connecticut in 1958. He received his Bachelor of Science degree in Electrical Engineering from the Massachusetts Institute of Technology in June of 1980, and his PhD degree in Electrical Engineering and Computer Sciences from the University of California, Berkeley in May 1985. In January 1985, Dr. DeMarco joined the faculty of the Department of Electrical and Computer Engineering at the University of Wisconsin-Madison, where he is currently an Associate Professor.

Claudio A. Cañizares (S'87) was born in Mexico, D.F. in 1960. In April 1984, he received the Electrical Engineer degree from the Escuela Politécnica Nacional (EPN), Quito-Ecuador, where he is currently an Assistant Professor on leave of absence, and the MSEE from the University of Wisconsin-Madison in 1988. Mr. Cañizares is a PhD student, T.A., and R.A. at the University of Wisconsin-Madison, sponsored by Fulbright, OAS, and EPN. 
Discussion

K. R. Padiyar (Indian Institute of Science, Bangalore, India): The authors of this paper are to be complimented for a novel approach to the security analysis of $\mathrm{AC} / \mathrm{DC}$ systems using a vector energy function.

The authors have rightly used the structure preserving energy function (SPEF) which enables the retention of converter buses with the modelling of the DC link as loads. In reference (1) of the paper, the loads were treated as constant current type and distribution factors were used to eliminate the converter buses by adding power injection at the generator internal nodes. However with the availability of SPEF it is not necessary to eliminate the converter buses [A]. Actually, we have observed that it is adequate to include only the term $V_{a c}^{d c}$ of the paper [B]. Incidentally it is to be noted that the first two terms of $V_{a c}^{d c}$ (equation (5) of the paper) are only approximations and the correct expressions are

$$
\int_{t_{o}}^{t} P_{r} \frac{d \delta_{r}}{d t} d t+\int_{t_{o}}^{t} P_{i} \frac{d \delta_{i}}{d t} \cdot d t
$$

(Note that $P_{r}$ and $P_{i}$ are not constants)

While it is natural to look for an additional term for the HVDC system in our view, the inclusion of $V_{d c}$ as given in the paper leads to numerical errors in the prediction of the critical clearing time. In this context, we would like the authors to give some more details regarding the computation of u.e.p and the system simulation. In our view, the computation of u.e.p with structure preserving model is problematic Also the use of PEBS in a non conservative system (the energy function is not constant along the post fault trajectory) gives inaccurate results. The simulation of a solid three phase fault at the converter bus in a transient stability program is likely to be inaccurate due to inadequate modelling of commutation failure and the response of the converter control.

We have the following comments and queries and would request the authors for their response.

1. The use of simplified converter control model does not imply that mode shifts cannot be considered. The so called 'performance' or 'response' type model for converter control can be quite accurate [C].

2. The assumption $\dot{I}_{d}>0$ cannot be justified as this implies that the DC current cannot decrease!

3. Normally in transient stability programs, the four valve conduction mode is not modelled although during a fault close to the converte bus, this converter will enter into a 3-4 valve conduction mode. But this is transient and the VDCOL will reduce the DC current. Actually for faults near the inverter bus, the commutation failure will interrupt the power flow for the duration of the fault. Also it is not clear as to why the converter mode change should prevent the computation of critical clearing time.

\section{References}

[A] K. R. Padiyar, "HVDC Power Transmission Systems-Technology and System Interactions," New Delhi, Wiley Eastern, 1991 pp. 208-209.

[B] K. R. Padiyar and H. S. Y. Sastry, "Direct Stability Analysis of AC/DC Power Systems Using A Structure Preserving Energy Function," Proceedings of IEEE Int. Conf. on Computer Systems and Signal Processing, Bangalore, India, Dec. 10-12, 1984.

[C] S. Lefebvre, et al., "Experience With Modeling MTDC Systems In Transient Stability Programs," IEEE Trans. on Power Delivery, vol. 6 , no. 1 , Jan. 1991 , pp. $405-413$.

C. L. DEMARCO AND C. A.CAÑIZARES: Professor Padiyar's discussion brings to light a very relevant work of his own in [1]. It is unfortunate that we were not aware of that publication when composing the current paper. One goal of this closure must therefore be to compare the current paper to [1]. As Professor Padiyar notes in his discussion, a key difference lies in our inclusion of an explicit term in the system energy function that is associated with state variables of a dynamic model for DC controllers, denoted as $V_{d c}$ in the paper. The treatment of the energy function terms associated with $\mathrm{AC}$ system behavior $\left(V_{\boldsymbol{x}}^{a c}\right)$ and with real and reactive power demands at inverter and rectifier buses $\left(V_{\alpha}^{d c}\right)$ is quite similar in both papers. Credit for the original observations on the role of these terms in a structure preserving energy function clearly belongs with Professor Padiyar. For simplicity of notation, the sum of $V_{a c}^{a c}$ and $V_{a c}^{d c}$ is denoted as $V_{a c}$ in the current paper, and this notation will be used in this closure.

Based on his work in [1], Professor Padiyar asserts that it is sufficient to include the only the $V_{a c}$ term in the energy based stability analysis, and goes on to state in his discussion that "inclusion of $V_{d c}$ as given in the paper leads to numerical errors in the prediction of the critical clearing time." This criticism goes to the very heart of the current paper, and if valid, would imply that the approach presented therein was unnecessary and perhaps erroneous. We must take issue with Professor Padiyar's assertion. His comment is no doubt based on his interpretation of the results reported in Table 1 of the paper, which uses the energy function to estimate clearing times for the very small ac/dc test system shown in Figure 6. The results reported in Table 1 do show that some clearing time estimates for this test case are rather poor. However, our experience does not support Professor Padiyar's conclusion that the problem lies in the construction of the system energy function. Instead, we believe the problem lies in the use of a constant energy contour evaluated by the controlling UEP or PEBS method to estimate the stability boundary. We offer the following evidence in support of our interpretation. First, the small test system constructed in Figure 6 of the paper is in many ways a worst case example for UEP and PEBS calculations, in the sense that all ac buses are extremely tightly coupled to the de link behavior. In such cases, our experience shows that one may have a system energy function that satisfies the basic properties of being positive definite about the stable equilibrium, and non-increaing along trajectories, and yet use of that function to estimate the stability boundary with either a controlling UEP or PEBS method can be very inaccurate. For example, in the controlling UEP method, the constant energy contour remains a good "fit" to the actual stability boundary only in a small neighborhood of the UEP. If the fault-on trajectory exits the stability region some distance from this point, the resulting clearing time estimate is poor. In our computational experience, the approximation of the stability boundary by the constant energy contour was made worse by neglecting the $V_{d c}$ term.

Fortunately, this type of very small test system with all ac buses tightly coupled to HVDC links is not commonly found in practice. As authors, we are perhaps at fault for presenting the work before more thorough simulation tests with larger, more realistic systems were conducted. In the text of the paper, we stated our belief that "the PEBS method applied to the proposed ac/dc TEF will produce good clearing time estimates for faults at least one or two buses away from the HVDC link." Since the paper's submission, test 
cases have been examined for a 173 bus system having two dc links, and these studies support our assertion in the paper. Table $\mathrm{C} 1$ shows clearing time estimates for several bus faults in this 173 bus study, using the total energy function that includes the $V_{d c}$ term. Complete details of this test system and studies are available in [2]. Note that the accuracy of clearing time estimates for these faults is within 7\%. Moreover, the cases shown include a bus fault close to HVDC converter buses; bus 159 fault is second neighbor to an inverter. While further studies would of course be useful, we wish to emphasize that these cases are representative of our numerical experience to date.

As a final point in support of the need for inclusion of the $V_{\alpha}$ term in an overall system energy function, the reader should consider the interpretation of Figure 5 of the paper. To use a system energy function for stability or security analysis, a key property is that it should, as nearly as possible, be non-increasing along post disturbance system trajectories. Figure 5 demonstrates a case in which use of the $V_{a c}$ alone would result in severe violation of this property, while use of the weighted sum of $V_{a c}$ and $V_{d c}$ yields a total system energy that is nearly decreasing throughout. As noted above, this small example is in many ways a worst case test. Figure $\mathrm{C} 1$ shows the behavior of the paper's total energy function for the 173 bus test system. For this test case, the energy is truly nonincreasing along the whole trajectory.

Hopefully our discussion above answers Professor Padiyar's point regarding the need for the $V_{d c}$ term. With this complete, we can address a number of other points raised in his discussion. With regard to his point (1), we agree that our criticism of simplified converter control models in the paper was indeed too strong, as these models can implicitly account for control mode switching in an accurate manner. Our goal was only to stress that the model of the paper treated such mode switching explicitly.

In point (2), the discusser notes that it is physically impossible for $\dot{I}_{d c}$ to always be greater than zero, as then the dc current could never decrease. However, our discussion on page 3 of the paper applied only to transient conditions when the link was under inverter control with the rectifier at its minimum firing angle, with the added idealized assumption of constant ac system voltages. This discussion was not intended to imply $\dot{I}_{d c}>0$ throughout the full range of operation.

In point (3), the discusser notes that most transient stability programs do not explicitly represent commutation failures, but nonthe-less are able to simulate the impact of such events on ac system stability. Certainly the discusser is correct that with such a simulation, critical clearing imes can be calculated. However, the paper took a conservative approach, and labeled commutation failure as being outside the range of validity of the dynamic model for the converters. Hence no attempt was made to ascertain whether or not the system returned to an acceptable operating point following commutation failure. This approach was justifiable for the security calculations illustrated in Figures 7-10, where the system is assumed to be in normal operation, and stability margins are estimated. However, the discusser is correct that the approach in the paper would need to be extended if the energy method was to be routinely used for clearing time estimates. To do so, one would represent such events by approximating the active and reactive power demands "seen" by the ac system at converter buses, with suitable modifications to the $V_{a c}^{d c}$ term in (5). This has not yet been tested.

Finally, to address a incidental point made by the discusser, he is also correct in his statement that the first two terms in equation (5) may be viewed as an approximation to a path dependent integral along trajectories. However, even an "exact" path dependent integral is not guaranteed to produce an energy function that is nonincreasing along trajectories, unless a particular (and computationally costly) choice of trajectories is made [3]. Most approaches using path dependent integrals in the literature only validate this property for a set of computational examples. As the paper performed this same type of computational validation for the terms in (5), the relation to path dependent integrals was not stressed.

Despite our need to take issue with one of the discusser's points, the authors are very grateful to Professor Padiyar for the insightful comments provided.

\section{References}

[1] K. R. Padiyar and H. S. Y. Sastry, "Direct Stability Analysis of AC/DC Power Systems using a Structure Preserving Energy Function," Proc. of IEEE Int. Conference on Computer Sys. and Signal proc., Bangalore, India, Dec. 10-12, 1984.

[2] C. A. Cañizares, "Voltage Collapse and Transient Energy Function Analyses of AC/DC Systems," Ph. D. Dissertation, Department of Electrical and Computer Engineering, University of Wisconsin-Madison, August, 1991.

[3] C. L. DeMarco, "An Optimal Control Interpretation of Path Dependent Energy Integrals in Power System Stability Analysis," to appear, Proc. of the 1992 American Control Conference, Chicago, IL, June 24-26, 1992.

\begin{tabular}{|c|c|c|}
\hline Fault Bus & $\begin{array}{c}\text { Clearing Time }\left(t_{c c}\right) \\
\text { Simulation Estimates }\end{array}$ & $\begin{array}{c}\text { Clearing Time } \\
\text { PEBS Estimates }\end{array}$ \\
\hline 12 & $0.020<t_{c c}<0.025 \mathrm{sec}$ & $0.025 \mathrm{sec}$ \\
\hline 51 & $0.060<t_{c c}<0.065 \mathrm{sec}$ & $0.069 \mathrm{sec}$ \\
\hline 98 & $0.060<t_{c c}<0.065 \mathrm{sec}$ & $0.067 \mathrm{sec}$ \\
\hline 159 & $0.035<t_{c c}<0.040 \mathrm{sec}$ & $0.039 \mathrm{sec}$ \\
\hline
\end{tabular}

Table C1: Clearing Time Estimates for Faults on 173 Bus Test System 


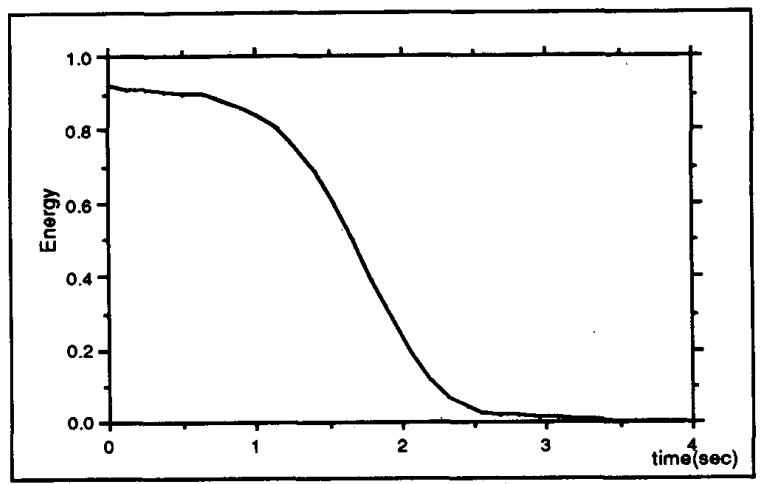

Figure C1: Decay of Total Energy along Trajectory from Unstable Equilibrium to Operating Point - 173 Bus Test System

Manuscript received April 8, 1992. 